\title{
Initial experience of the implementation of a cardio-oncology program for breast cancer patients receiving modern radiotherapy in a tertiary institution
}

\author{
Pei-Yu Hou ${ }^{1,2}$, Yen-Wen Wu ${ }^{3,4,5}$, Le-Jung Wu ${ }^{1}$, Chen-Hsi Hsieh ${ }^{1,5,6}$, Chen-Xiong Hsu ${ }^{1,2}$, Deng-Yu Kuo ${ }^{1}$, \\ Yueh-Feng Lu' ${ }^{1}$, Hui-Ju Tien ${ }^{1,2}$, Hsin-Pei Yeh ${ }^{1}$, Pei-Wei Shueng ${ }^{1,5,7}$, Bin S. Teh ${ }^{8}$ \\ ${ }^{1}$ Department of Radiation Oncology, Far Eastern Memorial Hospital, New Taipei City, Taiwan; ${ }^{2}$ Department of Biomedical Imaging and \\ Radiological Sciences, National Yang Ming Chiao Tung University, Taipei, Taiwan; ${ }^{3}$ Department of Nuclear Medicine, Far Eastern Memorial \\ Hospital, New Taipei City, Taiwan; ${ }^{4}$ Division of Cardiology, Cardiovascular Medical Center, Far Eastern Memorial Hospital, New Taipei City, \\ Taiwan; ${ }^{5}$ School of Medicine, National Yang Ming Chiao Tung University, Taipei, Taiwan; ${ }^{6}$ Institute of Traditional Medicine, School of Medicine, \\ National Yang Ming Chiao Tung University, Taipei, Taiwan; ${ }^{7}$ Department of Radiology, Far Eastern Memorial Hospital, New Taipei City, Taiwan; \\ ${ }^{8}$ Department of Radiation Oncology, Houston Methodist Hospital, Cancer Center and Research Institute, Weill Cornell Medical College, Houston, \\ TX, USA \\ Contributions: (I) Conception and design: PY Hou, PW Shueng; (II) Administrative support: All authors; (III) Provision of study materials or patients: \\ All authors; (IV) Collection and assembly of data: All authors; (V) Data analysis and interpretation: PY Hou, PW Shueng; (VI) Manuscript writing: \\ All authors; (VII) Final approval of manuscript: All authors. \\ Correspondence to: Pei-Wei Shueng. No. 21, Sec. 2, Nanya S. Rd., Banciao Dist., New Taipei City 220, Taiwan. Email: shuengsir@gmail.com; Bin S. \\ Teh. 6565 Fannin, Ste\#DB1-077 Houston, TX 77030, USA. Email: bteh@houstonmethodist.org.
}

Background: The modern era of multimodality cancer treatment strategies has contributed to a much longer survival time of breast cancer patients. Accordingly, cardiovascular toxicity related to anticancer therapy including radiation-induced heart disease has become an issue of concern. To address this issue, it is important to develop cardio-oncology programs to provide cardiovascular assessment, surveillance and management for patients receiving cancer therapy with potential heart impairment.

Methods: A multidisciplinary cardio-oncology combined care team and clinical protocol were established. Cardiac assessments of patients at risk before radiation, and surveillance and management of cardiotoxicity after completing cancer therapy were performed. A novel radiotherapy planning technique with completedirectional-complete block in helical tomotherapy was used to reduce cardiac exposure dose and limit heart damage. We analyzed the effect in the patients receiving this modern technique and cardio-oncology care.

Results: The number of breast cancer patients receiving cardio-oncology care increased annually, from $64(71.9 \%)$ in 2013 to 177 (81\%) in 2019. A series of cardiac assessments were performed, including cardiac biomarkers, electrocardiogram, echocardiogram, and/or myocardial perfusion imaging to evaluate the patient's cardiac risk or development of subsequent cardiotoxicity after cancer therapy. Among the 303 patients who were treated with the modern radiotherapy technique, 15 (5\%) were diagnosed with cardiovascular disease by the combined care program.

Conclusions: For the breast cancer patients receiving multimodality cancer treatment with modern radiotherapy in this study, the multidisciplinary cardio-oncology program of screening, prevention, management and follow-up protocols reduced cancer therapy-related cardiovascular toxicity, and improved the clinical outcomes and quality of life for long-term survivors.

Keywords: Cardio-oncology (CO); breast cancer; radiotherapy; cardiovascular toxicity

Received: 30 July 2021; Accepted: 07 December 2021; Published: 30 December 2021.

doi: $10.21037 /$ tro-21-20

View this article at: https://dx.doi.org/10.21037/tro-21-20 


\section{Introduction}

The World Health Organization reported that breast cancer exceeded lung cancer to become the most commonly diagnosed cancer in 2020, with an estimated 2,261,419 new cases (11.7\% of all sites). It was the fifth leading cause of cancer mortality in 2020, with an estimated 684,996 new deaths (6.9\% of all sites) (1). The epidemiology of cancer in the United States has shown a similar trend. The American Cancer Society reported 281,550 new cases of invasive breast cancer in women and 2,650 cases in men in 2021, and that breast cancer was the leading site of new cancer cases. In addition, from 2008 to 2017, the invasive female breast cancer incidence rate increased slightly, by $0.5 \%$ per year (2).

The epidemiology of cancer and cardiovascular disease (CVD) has been frequently explored. Previous studies have shown associations between CVD and future cancer due to shared risk factors, and revealed the extent of the co-occurrence of CVD and cancer (3). The Surveillance, Epidemiology, and End Results database includes more than 60,000 women diagnosed with breast cancer aged 66 years or older between 1992 and 2000, and an analysis of the database revealed that the number of patients who died of CVD increased proportionally year by year. About 10 years or more after the diagnosis of breast cancer, the number of CVD-related deaths exceeded that caused by breast cancer, and CVD was the leading cause of death among elderly female breast cancer patients (4).

In addition, Stoltzfus et al. reported a heart diseasespecific mortality rate of 10.61 per 10,000 person-years among patients with cancer, which was 2.24 times higher than the standardized rate for fatal heart disease [95\% confidence interval (CI): 2.23-2.25, $\mathrm{P}<0.0001]$ (5). Advances in breast cancer treatment have reduced the cancer mortality rate. Consequently, the increase in long-term cancer survivors has led to an increased overlap between patients with heart disease and cancer. Thus, awareness of cardiac toxicity and impaired cardiac function associated with cancer therapy is an increasingly important issue.

Cardiotoxic agents such as anthracycline-based chemotherapy, anti-human epidermal growth factor receptor 2 (HER2) target therapy, and breast radiotherapy (RT) are commonly used treatments for breast cancer. For patients with locally advanced breast cancer, regional nodal irradiation (RNI) including internal mammary chain (IMN) may be indicated to reduce mortality and recurrence $(6,7)$. However, the more extensive the RT volume, the greater the exposure dose to adjacent normal organs, including the heart and lungs. In left breast cancer patients, RTrelated cardiac toxicity has become an important issue, and an increasing number of patients are receiving novel immunotherapies. Associated cardiovascular (CV) toxicities such as myocarditis are also a concern. With breast cancer patients surviving longer, the incidence of heart disease increases due to age or late side effects of anticancer therapy. The incidence of breast cancer in Taiwan has shown a bimodal distribution, with most patients diagnosed between 45 and 65 years of age, which is 8 to 10 years earlier than in Western countries (8). Therefore, this patient population in Taiwan tend to have a longer survival duration, and late toxicity due to anticancer therapy is of particular concern due to potential adverse effects on clinical outcomes and quality of life.

Baseline CV risk assessments including serum cardiac biomarkers, electrocardiographic (ECG) and echocardiography or alternative cardiac imaging modalities are recommended for patients receiving potentially cardiotoxic cancer therapies by the European Society for Medical Oncology (ESMO) and American Society of Clinical Oncology (ASCO) $(9,10)$. In addition, the British Society of Echocardiography and British CardioOncology Society have published a protocol for baseline and surveillance echocardiography for cancer patients undergoing anthracycline treatment and/or anti-HER2 therapy (11). This protocol suggests for patients to have echocardiography before treatment, every 3 months during treatment, and 3-12 months after completing cancer therapy.

Currently, there is no consensus regarding the modality or frequency of screening and surveillance for $\mathrm{CV}$ toxicity caused by breast RT. As assessments before, during and after RT are needed, we cooperated with other specialists related to cancer and CVD management to initiate a cardiooncology $(\mathrm{CO})$ program and establish a clinical practice protocol at our tertiary institute. The purpose was to detect coexisting $\mathrm{CV}$ risk factors in breast cancer patients early, implement strategies for the prevention and treatment of CVD-related morbidity and mortality for either therapyrelated or patient-related cardiotoxicity. We also developed an RT planning technique to improve normal organ sparing and reduce potential toxicities, and reviewed the outcomes. We present the following article in accordance with the STROBE reporting checklist (available at https://dx.doi. org/10.21037/tro-21-20). 


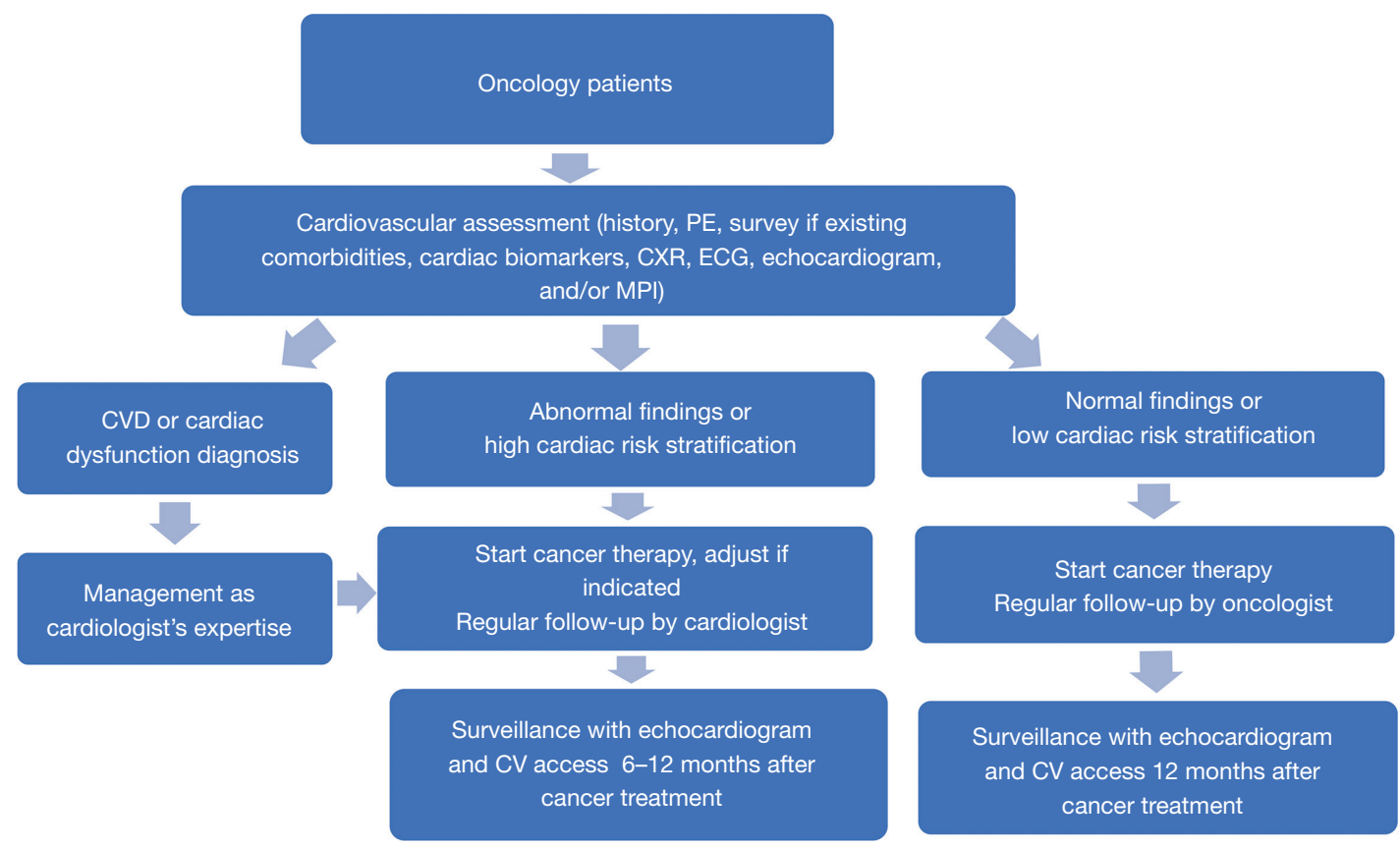

Figure 1 Protocol for patients receiving CO combined care, CV assessments and surveillance. PE, physical examination; CXR, chest X-ray; ECG, electrocardiograph; MPI, myocardial perfusion imaging; CVD, cardiovascular disease; CV, cardiovascular; CO, cardio-oncology.

\section{Methods}

\section{Establishment of the CO program and combined care protocol}

The radiation oncology department cooperated with cardiology, medical oncology and nuclear medicine departments to establish the CO team devoted to reducing cardiac impairment associated with cancer therapy. After patients had been enrolled into the CO program, they received a series of examinations for cardiac risk stratification, including history, survey of any existing comorbidities, physical examination, cardiac biomarkers troponin and $\mathrm{N}$-terminal pro-brain natriuretic peptide (NT-proBNP), chest X-ray (CXR), ECG, echocardiogram, and/or myocardial perfusion imaging (MPI). The aim was to evaluate the cancer patients' baseline cardiac function, screen for any underlying CVD or heart dysfunction, and assess the cardiac risk before performing RT, especially in left breast cancer patients. The anticancer treatment strategy may then have been adjusted according to the patient's cardiac risk stratification to avoid potential cardiotoxicity. The protocol for the patients undergoing CO combined care and surveillance is shown in Figure 1. If the patients had CVD, cardiac dysfunction, left ventricular ejection fraction (LVEF) $\leq 50 \%$, any symptom of heart failure, or elevated cardiac biomarkers troponin or NT-proBNP, then the patients would receive management as cardiologist's opinion before further cancer therapy. If the patients had preexisting CV risk factors of hypertension (HTN), diabetes mellitus (DM), dyslipidemia, cerebrovascular accident (CVA), obesity (body mass index $\geq 27$ ), or smoking, or other abnormal findings on $\mathrm{CV}$ assessment, but without diagnosis of CVD or cardiac dysfunction yet, they were stratified as high cardiac risk stratification, and would receive regular CV follow up after completion of cancer therapy with the cardiologist. If the patients had normal CV assessment and no associated $\mathrm{CV}$ risk factors, they were stratified as low risk and could have follow up after completion of cancer therapy with the oncologist. After completing cancer therapy, the patients were regularly followed up for malignancy and CV status to detect treatment-related CV toxicities early and allow for appropriate management to improve their clinical outcomes and quality of life.

Interdisciplinary discussions were routinely held to discuss the clinical practice of the CO program, referral protocol, personalized adjustment in cancer therapy strategy, associated research and education. Updated research data and medical knowledge were also provided to the team. The purpose of the CO team was to create a comprehensive, long-term care program for the cancer 


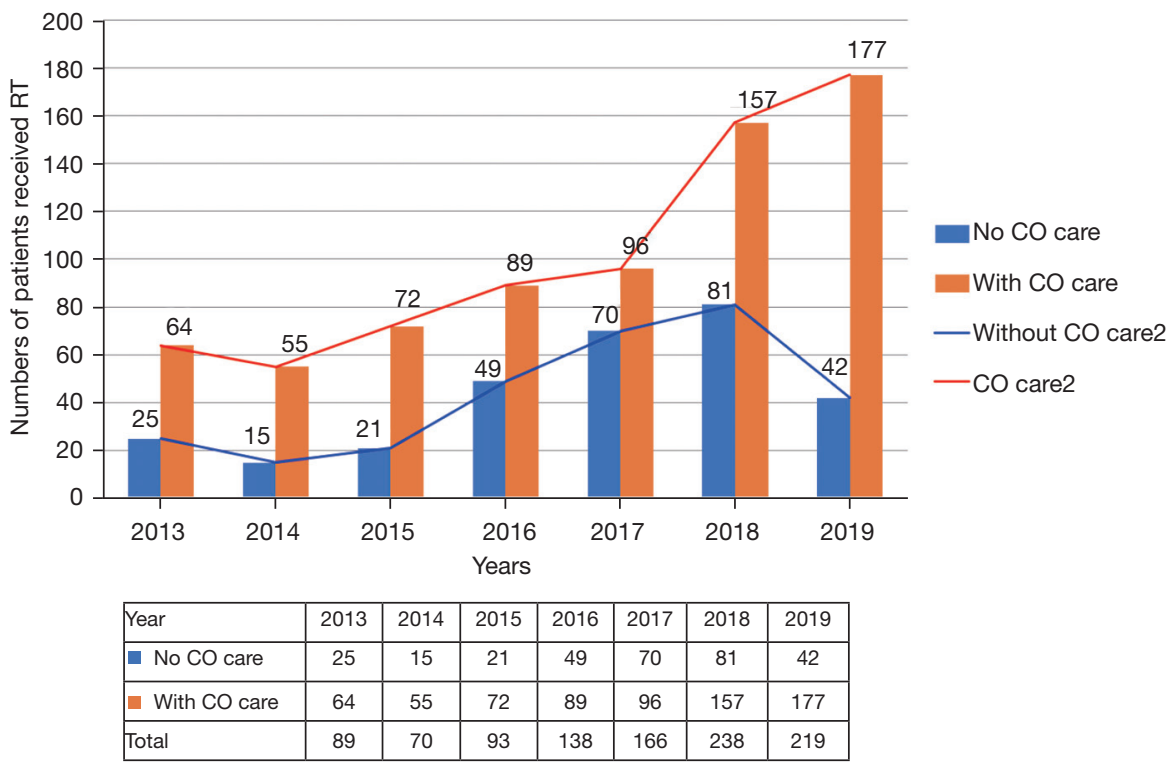

Figure 2 The increase in the number of breast cancer patients receiving CO combined care from 2013 to 2019. CO, cardio-oncology.

patients, increase treatment benefits and decrease potential side effects.

\section{Novel RT planning technique}

RT-induced CV impairment had been recognized. In 2014, we designed a directionally and completely blocked structure in helical tomotherapy (HT) (12). This technique successfully overcame the problem of extensive lowdose exposure to adjacent normal organs when using the radiation arc planning technique. The novel design reduced the dose to the heart, ipsilateral and contralateral lung, and contralateral breast, and also achieved good dose coverage and conformity for left-sided breast RT (12). In 2020, based on the previous technique, we developed an improved complete-directional-complete block (CDCB) technique in HT for left breast cancer patients receiving RNI with IMN. This CDCB technique with optimal restricted angle in HT achieved good RT target volume coverage, dose homogeneity and conformity, and allowed sparing of the heart, left anterior descending artery and bilateral lungs. Furthermore, this CDCB technique was shown to further reduce RT-related $\mathrm{CV}$ and lung toxicities (13). As the evaluation of RT planning, the constraints of organs at risk were a mean heart dose $\leq 20 \mathrm{~Gy}, \mathrm{~V} 25<10 \%$, a mean lung dose $\leq 20$ Gy, V $20 \leq 35 \%$.

The study was conducted in accordance with the
Declaration of Helsinki (as revised in 2013). The study was approved by the human experimentation committee of the Far Eastern Memorial Hospital (No. 109107-F) and individual consent for this retrospective analysis was waived.

\section{Statistical analysis}

The research did not use a statistical analysis because it was the report of the clinical experience in an institution.

\section{Results}

\section{Improvement in clinical practice}

Since 2013, radiation oncologists concerned about radiation-induced heart disease (RIHD) actively referred cancer patients to our $\mathrm{CV}$ clinic before undergoing chest RT for associated cardiac risk assessments. The number of patients receiving $\mathrm{CO}$ combined care before breast RT increased annually from $64(71.9 \%)$ in 2013 to 177 (81\%) in 2019 (Figure 2). Through this multidisciplinary team work, more cancer patients received comprehensive care during the periods of cancer treatment and follow-up. Due to this success, we further promoted the protocol to interrelated cancer management specialists, and a formal multidisciplinary CO team was founded in 2020. The team members included radiation oncologists, cardiologists, medical oncologists, nuclear medicine radiologists and 


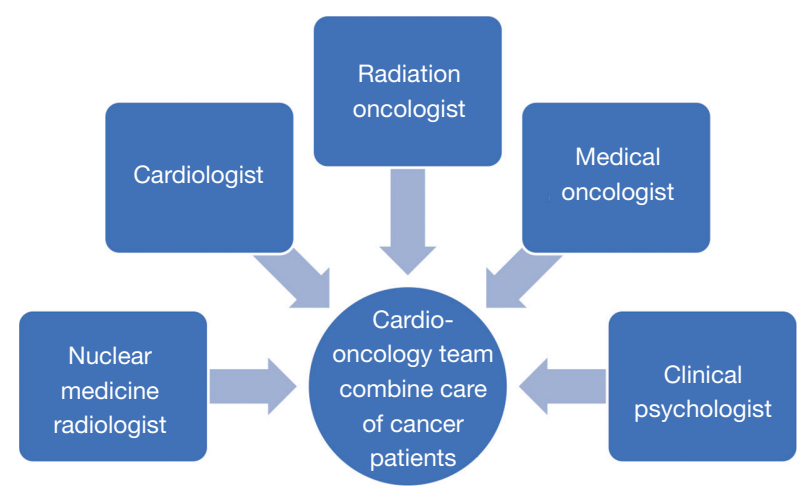

Figure 3 The cardio-oncology team. The multidisciplinary combined care team members included radiation oncologists, cardiologists, medical oncologists, nuclear medicine radiologists and clinical psychologists. CO, cardio-oncology.

clinical psychologists (Figure 3). Breast cancer patients at our institution now routinely receive $\mathrm{CO}$ combined care before systemic therapy or RT. We identify which patients are at an increased risk of cardiac dysfunction through baseline screening, and with early detection we can provide preventive strategies to reduce risks and manage existing clinical or subclinical CVD. After completing cancer therapy, long-term follow-up for cancer and CV status can reduce the morbidity and mortality of associated malignancy or $\mathrm{CV}$ disease.

\section{Patients' outcomes of CO care and novel RT technique}

From 2014 to 2020, we used the advanced breast RT techniques of complete block or CDCB in HT to minimize adjacent normal organ exposure and protect the heart. A total of 303 consecutive patients treated with this technique and were also enrolled into the $\mathrm{CO}$ combined care program and received cardiac evaluations. The median follow-up was 4.54 years (range, $1.2-7.75$ years). The median age was $55 \mathrm{y} / \mathrm{o}$ (range, $27-87 \mathrm{y} / \mathrm{o}$ ). The median BMI was 24.3 (range, 16.4-40.5). There were $56.6 \%$ patients with preexisting CVD or CV risk factors of HTN, DM, dyslipidemia, CVA, obesity, or smoking. The median LVEF revealed by baseline echocardiogram was $69 \%$ (range, $47-86 \%$ ). Through CV assessments by the $\mathrm{CO}$ team during or after cancer therapy, 15 patients $(5 \%)$ were diagnosed with CVD, including heart failure $(H F)(n=9)$, coronary artery disease $(n=3)$, and unspecified hypertensive cardiovascular disease $(\mathrm{n}=5)$ (Table 1). Ten patients were referred by radiation oncologists and were diagnosed with CVD before initiating breast RT.
Five of these patients received anthracycline chemotherapy and three received anti-HER2 target therapy before irradiation. For the two patients without systemic therapy, one was diagnosed with $\mathrm{HF}$ through $\mathrm{CV}$ assessments before RT, and the other developed hypertensive cardiovascular disease 14 months after completing RT. Most of these 15 patients had preexisting underlying diseases or $\mathrm{CV}$ risks including hypertension, dyslipidemia, diabetes mellitus, cerebrovascular accident, obesity, or smoking. The only patient without patient-related risk factors stratified as low risk group developed HF after anthracycline and trastuzumab treatment. Due to the incidence of $\mathrm{CV}$ comorbidities among the cancer patients, the application of the novel RT technique to reduce heart dose was an important factor to improve the clinical outcomes. At our institution, for patients received left-breast RT with regional nodal irradiation, the heart mean doses of 4.27 Gy was comparable with systematic review data on modern RT techniques (14).

\section{Discussion}

Multidisciplinary approach is crucial in the management of CVD or cardiotoxicity for oncology patients. Our CO team was established to provide comprehensive $\mathrm{CV}$ care for cancer patients with a relatively high incidence of $\mathrm{CV}$ comorbidities receiving potentially toxic cancer therapy. It aimed to survey and detect CVD early, reduce and manage cardiotoxicity, improve clinical outcomes and quality of life, and provide long-term surveillance for cancer survivors. In addition, the team developed a protocol of clinical care, management guidelines, education for the attending members and clinical research.

At our institution, patients are enrolled into the CO combined care program due to concerns of RIHD, and undergo $\mathrm{CV}$ risk assessments just before receiving breast RT. However, many of these patients receive potentially cardiotoxic systemic treatments such as anthracycline or anti-HER 2 target therapy. In this study, $5.3 \%$ of the patients had CVD diagnosed through the $\mathrm{CV}$ assessments according to the CO protocol. Half of them were diagnosed after completing chemotherapy with/without anti-HER2 therapy. If they had received $\mathrm{CO}$ combined care before initiating any treatment that could potentially damage the heart, prevention strategies such as angiotensin-converting enzyme inhibitor, angiotensin receptor blocker or selected beta blocker treatment $(15,16)$ or other treatments could have been given to avoid the development and progression 


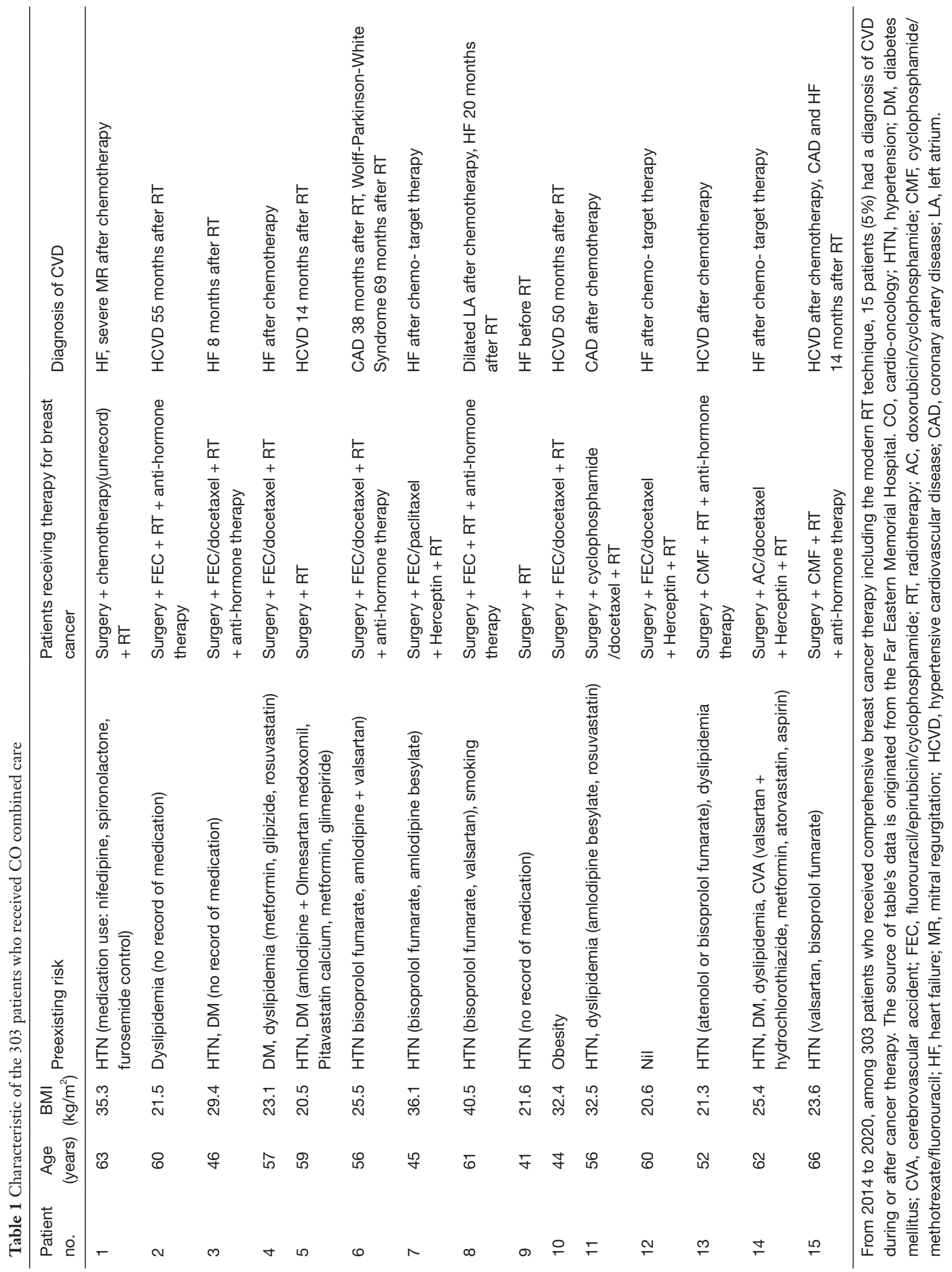


of cardiotoxicity. In addition, adjustments to cancer therapy associated with $\mathrm{CV}$ toxicity could have been considered. Providing appropriate care for patients could reduce possible cardiac dysfunction, avoid the discontinuation of cancer therapy, and improve treatment effects. Therefore, initiating $\mathrm{CO}$ care as early as possible is necessary, especially when planning to deliver potentially cardiotoxic cancer treatment. LVEF assessment is helpful to define cardiac function status so that the cardiotoxic therapy can be adjusted, although there are variable definitions of cardiac dysfunction according to an abnormal or decreased LVEF value. In general, if a patient has an LVEF $>50 \%$ and no symptoms of heart failure, cancer therapy can be initiated. On the other hand, in patients with an LVEF $<40 \%$, potentially cardiotoxic therapy with anthracycline agents or anti-HER2 therapy should be withheld.

The history of CO dated back in 1967 when a case of anthracycline-induced cardiotoxicity was first reported (17). Three decades later, the term CO was mentioned by Cardinale in 1996 (18). These studies highlighted cardiac damage induced by cancer therapy. Anti-HER2 target therapy-induced cardiotoxicity has been reported since its registration by the Food and Drug Administration in 1998 (19,20). For RIHD, an increased relative risk of cardiac events has also been reported. Darby reported a proportional increase in the association between major cardiac event rates and mean heart dose by $7.4 \%$ per Gy without threshold (21). The heart exposure dose has also been associated with an increase in cardiac mortality even with modern RT (14). The cardiac exposure indicated a 0.04 excess rate ratio per Gy whole heart dose for cardiac mortality incidence more than 10 years after RT. RTinduced cardiac damage includes vascular endothelium damage, valvular disease, pericarditis, cardiomyocyte damage, conduction dysfunction and heart failure. If patients need to receive chest RT, a precise RT field and delivery using intensity modulated RT (22) or deepinspiration breath holding should be encouraged to reduce the heart dose. Patients undergoing left breast or chest RT have been demonstrated to have higher rates of major cardiac toxicity than those undergoing right-side RT, and particular care should be paid to existing CVD risk factors (23-29). In cases of a complex RT treatment volume such as with RNI including IMN irradiation for left breast cancer, the use of a modern RT technique to optimize dose conformity, target coverage and spare OARs can be helpful in protecting normal organs without compromising disease control (13,30-34). At our institution, we use novel rotational RT techniques with $\mathrm{HT}$ or volumetric modulated arc therapy for left breast cancer patients receiving complex RNI treatment volume. From 2017 to 2020, the average heart mean dose of 108 patients was 4.27 Gy. The heart sparing effect with our novel RT technique was promising.

The ASCO Practice Guidelines clarified which cancer patients are at an increased risk of developing cardiac dysfunction. The lifetime cumulative dose of anthracycline agents with doxorubicin $\geq 250 \mathrm{mg} / \mathrm{m}^{2}$ or equivalent dose, sequential anthracycline and trastuzumab therapy, chest RT $\geq 30$ Gy including the heart in the radiation field, or lowerdose anthracycline treatment (e.g., doxorubicin $<250 \mathrm{mg} / \mathrm{m}^{2}$, epirubicin $<600 \mathrm{mg} / \mathrm{m}^{2}$ ) in combination with lowerdose RT ( $<30 \mathrm{~Gy}$ ) are therapy-related risk factors used to identify cardiotoxicity (10). Echocardiography is the preferred tool for diagnostic workup of surveillance and monitoring during and after cancer therapy in patients at risk of cardiac dysfunction. After completing cancer treatment, cardiac echo surveillance should be performed every 6 to 12 months. Cardiac magnetic resonance imaging or multigated acquisition (MUGA) are alternatives if echocardiography is unavailable or not feasible. Monitoring serum cardiac biomarkers (troponins, natriuretic peptides) is also recommended. The ESMO consensus on CV toxicity related to malignancy or therapy provided guidelines for the prevention, methods, tools and frequency of screening and monitoring and managing existing CVD in cancer patients throughout oncological treatment. The guidelines focused on specific chemotherapy agents, anti-HER2 target therapy and left-sided chest or mediastinal RT that may substantially affect the CV system. Close collaboration between multidisciplinary groups was also suggested (9). However, the screening, surveillance approaches and frequency of monitoring $\mathrm{CV}$ status were not mentioned in the consensus. As more international training programs and practice guidelines had been established, we could follow a role model to build up a suitable program.

Interdisciplinary communication is important and affects the efficiency and execution of team work. When we implemented the CO program at our institute, all breast cancer patients were actively referred to $\mathrm{CV}$ specialists for screening before receiving cancer therapy and then received surveillance after completing treatment. However, if CO combined care is offered to patients with other kinds of malignancy undergoing potentially cardiotoxicity therapy, there will be an increase in the number of patients enrolled in the CO care program. Therefore, we recommend establishing a standardized protocol that 
can be applied clinically to assess the CV risk and clearly define cardiotoxicity or subclinical cardiotoxicity. Thus, the associated specialist including medical oncologists and radiation oncologists can perform preliminary screening and assessment the $\mathrm{CV}$ risk first. Then, only the high-risk individuals need be referred to help the team focus on the susceptible patients. Adapting the surveillance approach and frequency according to risk stratification would be expected to reduce the frequency or even omit long-term follow-up in selected very low-risk patients. Bouwer et al. investigated cardiotoxicity during long-term trastuzumab use in breast cancer patients, and found that in nonsmoking patients with baseline LVEF $>60 \%$ and without cardiotoxicity during trastuzumab treatment, the cumulative incidence of severe cardiotoxicity was low, and that serial cardiac monitoring could be omitted safely (35). Thus, using current multiple treatment modalities related to $\mathrm{CV}$ toxicity, a CO team could identify who does or does not need cardiac monitoring, and improve the use of clinical resources.

In addition to the aforementioned $\mathrm{CV}$ toxicities, other CV-related side effects in cancer patients have been reported. A Danish population-based cohort study confirmed the relationship between cancer and arterial thromboembolism (ATE), which was defined as the composite of myocardial infarction, ischemic/unspecified stroke, and peripheral arterial occlusion. Cancer patients were at an increased risk of ATE. Six months after a cancer diagnosis, the cumulative incidence for ATE was two-fold higher in the cancer group, and this increase was sustained for 12 months following the cancer diagnosis. ATE was also associated with increased mortality among cancer patients (hazard ratio 3.28). Physicians should be aware of this important risk (36). Further novel target therapies and immunotherapies associated with cardiac side effects can be expected. Such treatment should be developed taking CV toxicity into account.

To develop and maintain a CO program, comprehensive associated resources are needed. Although this may be possible at larger hospitals or medical centers, it may not be possible at regional or district hospitals. Our tertiary institution experience could be reproduced and promoted to other hospitals. To improve medical treatment in Taiwan, our protocol could be followed to screen cancer patients initially. If patients have existing $\mathrm{CV}$ risk factors or are shown to be at risk, then they can be referred to institutions with $\mathrm{CO}$ teams to receive comprehensive $\mathrm{CO}$ care. The limitation of this study was that lack of a control population with regard to the CVD incidence. The period of follow up was relatively short in considering of the development of cardiotoxicity. The effort of cardio-oncology care might be more convinced as longer period of follow up.

\section{Conclusions}

A CO program can provide comprehensive $\mathrm{CV}$ care for cancer patients with a relatively high incidence of $\mathrm{CV}$ comorbidities who are scheduled to receive potentially cardiotoxic cancer therapy. For breast cancer patients, the promotion of screening, early detection, prevention, management and surveillance of cardiotoxicity can reduce possible cancer therapy-related CV impairment, and improve clinical outcomes and the quality of life for longterm survivors.

\section{Acknowledgments}

Funding: This work was supported by the Far Eastern Memorial Hospital (FEMH-2021-C-057).

\section{Footnote}

Provenance and Peer review: This article was commissioned by the editorial office, Therapeutic Radiology and Oncology for the series "Cardio-Oncology". The article has undergone external peer review.

Reporting Checklist: The authors have completed the STROBE reporting checklist. Available at https://dx.doi. org/10.21037/tro-21-20

Data Sharing Statement: Available at https://dx.doi. org/10.21037/tro-21-20

Conflicts of Interest: All authors have completed the ICMJE uniform disclosure form (available at https://dx.doi. org/10.21037/tro-21-20). The series "Cardio-Oncology" was commissioned by the editorial office without any funding or sponsorship. YWW and PWS served as the unpaid Guest Editors of the series. CHH serves as the unpaid editorial board members of Therapeutic Radiology and Oncology from May 2020 to April 2022. The authors have no other conflicts of interest to declare.

Ethical Statement: The authors are accountable for all aspects of the work in ensuring that questions related 
to the accuracy or integrity of any part of the work are appropriately investigated and resolved. The study was conducted in accordance with the Declaration of Helsinki (as revised in 2013). The study was approved by the human experimentation committee of the Far Eastern Memorial Hospital of (No. 109107-F) and individual consent for this retrospective analysis was waived.

Open Access Statement: This is an Open Access article distributed in accordance with the Creative Commons Attribution-NonCommercial-NoDerivs 4.0 International License (CC BY-NC-ND 4.0), which permits the noncommercial replication and distribution of the article with the strict proviso that no changes or edits are made and the original work is properly cited (including links to both the formal publication through the relevant DOI and the license). See: https://creativecommons.org/licenses/by-nc-nd/4.0/.

\section{References}

1. Sung H, Ferlay J, Siegel RL, et al. Global Cancer Statistics 2020: GLOBOCAN Estimates of Incidence and Mortality Worldwide for 36 Cancers in 185 Countries. CA Cancer J Clin 2021;71:209-49.

2. Cancer Facts \& Figures 2021. Available online: https:// www.cancer.org/content/dam/cancer-org/research/cancerfacts-and-statistics/annual-cancer-facts-and-figures/2021/ cancer-facts-and-figures-2021.pdf

3. Lau ES, Paniagua SM, Liu E, et al. Cardiovascular Risk Factors are Associated with Future Cancer. JACC CardioOncol 2021;3:48-58.

4. Patnaik JL, Byers T, DiGuiseppi C, et al. Cardiovascular disease competes with breast cancer as the leading cause of death for older females diagnosed with breast cancer: a retrospective cohort study. Breast Cancer Res 2011;13:R64.

5. Stoltzfus KC, Zhang Y, Sturgeon K, et al. Fatal heart disease among cancer patients. Nat Commun 2020;11:2011.

6. Poortmans PM, Weltens C, Fortpied C, et al. Internal mammary and medial supraclavicular lymph node chain irradiation in stage I-III breast cancer (EORTC 22922/10925): 15-year results of a randomised, phase 3 trial. Lancet Oncol 2020;21:1602-1610. Erratum in: Lancet Oncol 2021;22:e5.

7. Whelan TJ, Olivotto IA, Parulekar WR, et al. Regional Nodal Irradiation in Early-Stage Breast Cancer. N Engl J Med 2015;373:307-16.
8. Health Promotion Administration Ministry of Health and Welfare, Taiwan. Available online: https://www.hpa.gov. tw/Pages/Detail.aspx? nodeid=269\&pid=13498

9. Curigliano G, Lenihan D, Fradley M, et al. Management of cardiac disease in cancer patients throughout oncological treatment: ESMO consensus recommendations. Ann Oncol 2020;31:171-90.

10. Armenian SH, Lacchetti C, Barac A, et al. Prevention and Monitoring of Cardiac Dysfunction in Survivors of Adult Cancers: American Society of Clinical Oncology Clinical Practice Guideline. J Clin Oncol 2017;35:893-911.

11. Dobson R, Ghosh AK, Ky B, et al. BSE and BCOS Guideline for Transthoracic Echocardiographic Assessment of Adult Cancer Patients Receiving Anthracyclines and/or Trastuzumab. JACC CardioOncol 2021;3:1-16.

12. Shiau AC, Hsieh CH, Tien HJ, et al. Left-sided whole breast irradiation with hybrid-IMRT and helical tomotherapy dosimetric comparison. Biomed Res Int 2014;2014:741326.

13. Yeh HP, Huang YC, Wang LY, et al. Helical tomotherapy with a complete-directional-complete block technique effectively reduces cardiac and lung dose for left-sided breast cancer. Br J Radiol 2020;93:20190792.

14. Taylor C, Correa C, Duane FK, et al. Estimating the risks of breast cancer radiotherapy: evidence from modern radiation doses to the lungs and heart and from previous randomized trials. J Clin Oncol 2017;35:1641-9.

15. Cardinale D, Colombo A, Sandri MT, et al. Prevention of high-dose chemotherapy-induced cardiotoxicity in highrisk patients by angiotensin-converting enzyme inhibition. Circulation 2006;114:2474-81.

16. Kalay N, Basar E, Ozdogru I, et al. Protective effects of carvedilol against anthracycline-induced cardiomyopathy. J Am Coll Cardiol 2006;48:2258-62.

17. Tan C, Tasaka H, Yu KP, et al. Daunomycin, an antitumor antibiotic, in the treatment of neoplastic disease. Clinical evaluation with special reference to childhood leukemia. Cancer 1967;20:333-53.

18. Cardinale D. A new frontier: cardio-oncology. Cardiologia 1996;41:887-91.

19. de Azambuja E, Bedard PL, Suter T, et al. Cardiac toxicity with anti-HER-2 therapies-what have we learned so far? Target Oncol 2009;4:77-88.

20. Florido R, Smith KL, Cuomo KK, et al. ardiotoxicity From Human Epidermal Growth Factor Receptor-2 (HER2) Targeted Therapies. J Am Heart Assoc 2017;6:e006915.

21. Darby SC, Ewertz M, McGale P, et al. Risk of ischemic heart disease in women after radiotherapy for breast 
cancer. N Engl J Med 2013;368:987-98.

22. Teh BS, Lu HH, Sobremonte S, et al. The potential use of intensity modulated radiotherapy (IMRT) in women with pectus excavatum desiring breast-conserving therapy. Breast J 2001;7:233-9.

23. Correa CR, Litt HI, Hwang W', et al. Coronary artery findings after left-sided compared with right-sided radiation treatment for early-stage breast cancer. J Clin Oncol 2007;25:3031-7.

24. Jagsi R, Griffith KA, Koelling T, et al. Rates of myocardial infarction and coronary artery disease and risk factors in patients treated with radiation therapy for early-stage breast cancer. Cancer 2007;109:650-7.

25. Hooning MJ, Botma A, Aleman BM, et al. Long-term risk of cardiovascular disease in 10-year survivors of breast cancer. J Natl Cancer Inst 2007;99:365-75.

26. Gutt R, Correa CR, Hwang WT, et al. Cardiac morbidity and mortality after breast conservation treatment in patients with early-stage breast cancer and preexisting cardiac disease. Clin Breast Cancer 2008;8:443-8.

27. Harris EE, Correa C, Hwang WT, et al. Late cardiac mortality and morbidity in early-stage breast cancer patients after breast-conservation treatment. J Clin Oncol 2006;24:4100-6.

28. Verma V, Fakhreddine MH, Haque W, et al. Cardiac mortality in limited-stage small cell lung cancer. Radiother Oncol 2018;128:492-7.

29. Haque W, Verma V, Haque A, et al. Trends in cardiac mortality in women with ductal carcinoma in situ. Breast Cancer Res Treat 2017;161:345-51.

30. Tyran M, Mailleux H, Tallet A, et al. Volumetricmodulated arc therapy for left-sided breast cancer and all regional nodes improves target volumes coverage and

doi: $10.21037 /$ tro-21-20

Cite this article as: $\mathrm{Hou} \mathrm{PY}, \mathrm{Wu} \mathrm{YW}, \mathrm{Wu} \mathrm{LJ}, \mathrm{Hsieh} \mathrm{CH}$, Hsu CX, Kuo DY, Lu YF, Tien HJ, Yeh HP, Shueng PW, Teh BS. Initial experience of the implementation of a cardiooncology program for breast cancer patients receiving modern radiotherapy in a tertiary institution. Ther Radiol Oncol 2021;5:21. reduces treatment time and doses to the heart and left coronary artery, compared with a field-in-field technique. J Radiat Res 2015;56:927-37.

31. Popescu CC, Olivotto IA, Beckham WA, et al. Volumetric modulated arc therapy improves dosimetry and reduces treatment time compared to conventional intensitymodulated radiotherapy for locoregional radiotherapy of left-sided breast cancer and internal mammary nodes. Int J Radiat Oncol Biol Phys 2010;76:287-95.

32. Nobnop W, Phakoetsuk P, Chitapanarux I, et al. Dosimetric comparison of TomoDirect, helical tomotherapy, and volumetric modulated arc therapy for postmastectomy treatment. J Appl Clin Med Phys 2020;21:155-62.

33. Lauche O, Kirova YM, Fenoglietto P, et al. Helical tomotherapy and volumetric modulated arc therapy: New therapeutic arms in the breast cancer radiotherapy. World J Radiol 2016;8:735-42.

34. Zhang Q, Yu XL, Hu WG, et al. Dosimetric comparison for volumetric modulated arc therapy and intensitymodulated radiotherapy on the left-sided chest wall and internal mammary nodes irradiation in treating postmastectomy breast cancer. Radiol Oncol 2015;49:91-8.

35. Bouwer NI, Steenbruggen TG, van Rosmalen J, et al. Cardiotoxicity during long-term trastuzumab use in patients with HER2-positive metastatic breast cancer: who needs cardiac monitoring? Breast Cancer Res Treat 2021;186:851-62.

36. Mulder FI, Horváth-Puhó E, van Es N, et al. Arterial Thromboembolism in Cancer Patients: A Danish Population-Based Cohort Study. JACC CardioOncol 2021;3:205-18. 